\title{
Spinopelvic Alignment Parameters in Spondylolisthesis Patients Compared to Nonspondylolisthesis Patients
}

\author{
Babak Alijani ${ }^{1}$ Armin Ramzannejad ${ }^{1}$ Shahrokh Yousefzadeh-Chabok ${ }^{2,3}$ Hamid Behzadnia \\ Mohammadreza Emamhadi ${ }^{1}$ Ali Davoudi-Kiakalayeh ${ }^{3}$ Enayatollah Bijani ${ }^{1}$ \\ ${ }^{1}$ Department of Neurosurgery, Poursina Hospital, Guilan University \\ of Medical Sciences, Rasht, Iran \\ 2 Neuroscience Research Center, Department of Neurosurgery, \\ Poursina Hospital, School of Medicine, Guilan University of Medical \\ Sciences, Rasht, Iran \\ ${ }^{3}$ Guilan Road Trauma Research Center, Guilan University of Medical \\ Sciences, Rasht, Iran \\ Indian J Neurosurg 2019;8:8-12

\begin{abstract}
Address for correspondence Hamid Behzadnia, MD, Guilan Road Trauma Research Center, Poursina Hospital, Namjoo Street, Rasht, Guilan 41937-13194, Iran (e-mail: artauth2016@gmail.com).
\end{abstract}

\begin{abstract}
Keywords

- spondylolisthesis

- spinopelvic

Objective We aimed at describing spin pelvic alignment features in patients with degenerative spondylolisthesis (DS), in comparison with other patients complaining of low back pain but no evidence of spondylolisthesis on standard standing lateral lumbosacral X-ray.

Methods In this prospective descriptive study, patients with low back pain included in two groups of DS and non-DS patients, according to preoperative lumbosacral X-ray in standard standing lateral position. Patient's demographic characteristics, as well as spinopelvic alignment parameters including pelvic incidence (PI), pelvic tilt (PT), sacral slope (SS), lumbar lordosis, and facet joint orientation, were collected. DS patients were compared with non-DS, age-matched control group.

Results DS patients were significantly obese $(p=0.031)$ and had a high PI (56.39 \pm 11.5$)$, SS (38.28 \pm 10.32$)$, and PT $(18.52 \pm 9.11)$, $(p=0.00,0.01,0.04$, respectively). Moreover, segmental lordosis at L2-L3, L3-L4, and L5-S1 levels demonstrated a significant increase $(p<0.05)$. Facet joints at $L 4-L 5$ level were located more sagittally ( $37.5 \pm 7.07$ vs. $40.71 \pm 6.33$ ).

- alignment

Conclusion PI, SS, and PT significantly increase in DS patients compared with non-

- parameters

- body mass index DS group. Elevated segmental lordosis at high lumbar levels and sagittal orientation of facet joints were other features observed in DS patients.
\end{abstract}

\section{Introduction}

Spinopelvic morphology and orientation balance the mechanical stress over lumbosacral junction. ${ }^{1}$ L5 slippage over S1, leading to L5-S1 spondylolisthesis disarranges sacropelvic orientation, resulting in sagittal imbalance of the spine. ${ }^{1}$ This condition is more frequent among women and patients younger than 50 years. ${ }^{2}$ Wiltse and Newman described five groups of patients: dysplastic, isthmic, traumatic, pathologic, and degenerative categories. Degenerative form of the disease involves L4-L5 level more frequently, despite other forms of spondylolisthesis that are more prevalent at L5-S1 level. ${ }^{2,3}$ Multiple pathologic factors have been described including elevated body mass index (BMI), facet joint osteoarthritis, ${ }^{4}$ incompetence of the paraspinal muscles and ligaments, and effect of female sexual hormones. ${ }^{5}$ Spondylolisthesis leads to decreased capacity of compensatory balance mechanism. ${ }^{2}$ Untreated patients develop structural abnormalities in vertebrae, adjacent nerve roots and soft tissue, reflecting as low back pain along with radicular neurologic deficit and sagittal pelvic imbalance. ${ }^{6,7}$ In recent few years, the presence of high pelvic incidence (PI) and sacral slope (SS) have been
DOI https://doi.org/

10.1055/s-0039-1695671

ISSN 2277-954X.
License terms

()(1) $\Theta \circledast$ 
highlighted in the setting of degenerative spondylolisthesis (DS). With focus on predisposing role of these factors, ${ }^{8,9}$ increasing pelvic tilt (PT) reimburses high PI as a compensatory mechanism..$^{9,10}$

Concomitance of overweight and vertical displacement of S1 endplate leads to anterior slippage of L4 over L5. The purpose of this study was to evaluate radiographic spinopelvic parameters and demographic features in patients diagnosed with DS, compared with the same findings in another group of patients with low back pain but no evidence of DS on X-ray.

\section{Materials and Methods}

This study was designed as prospective descriptive and observational case series. Institutional review board approved our study. After obtaining written consent form, we defined the two groups of patients referring to the clinic according to their basal spine X-ray in standard standing position. All of them had complaints of low back pain. The first group included 60 patients with DS, and the second consisted of 60 patients with low back pain due to other causes. We described the exclusion criteria as developmental spondylolisthesis, previous history of spinal neoplasm or fractures, and past spinal surgical interventions with or without prosthetic material deployment. We recorded BMI (weight [kg] divided by height $\left[\mathrm{m}^{2}\right]$ ), sex, and age of all patients. On lateral lumbosacral X-ray, we measured PT, SS, and PI ( - Fig. 1). Furthermore, we applied Digimizer software (v4.1.10; MedCalc Software) for calculating L1-S1 lordosis and segmental lordosis at levels of L1-L2 ( - Fig. 2) to L5-S1 ( $>$ Fig. 3), between the superior endplate of the upper vertebra and inferior endplate of the lower vertebra on X-ray, as well as facet joint orientation at levels

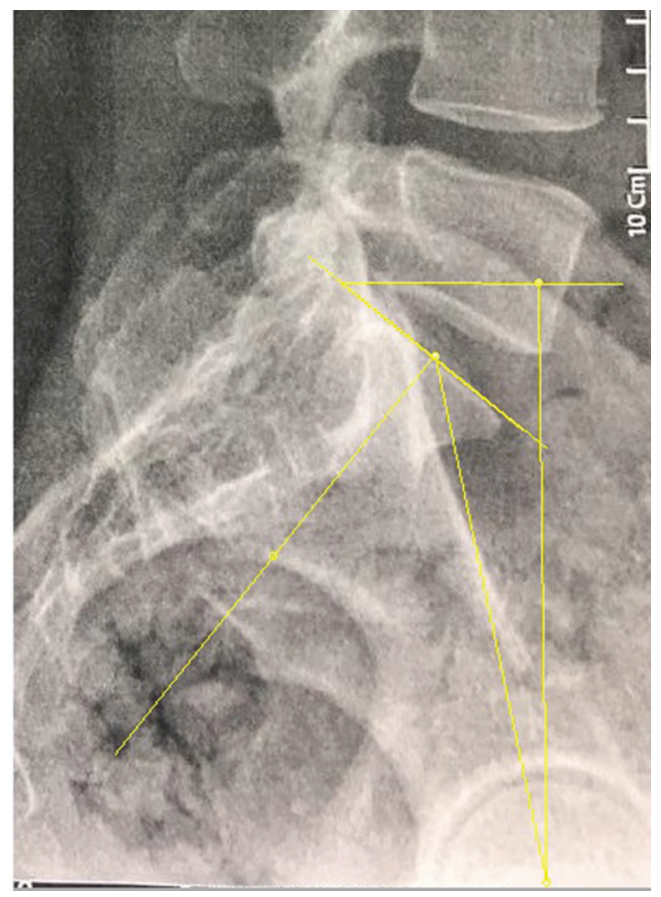

Fig. 1 Measurement of PI, SS, and PT.

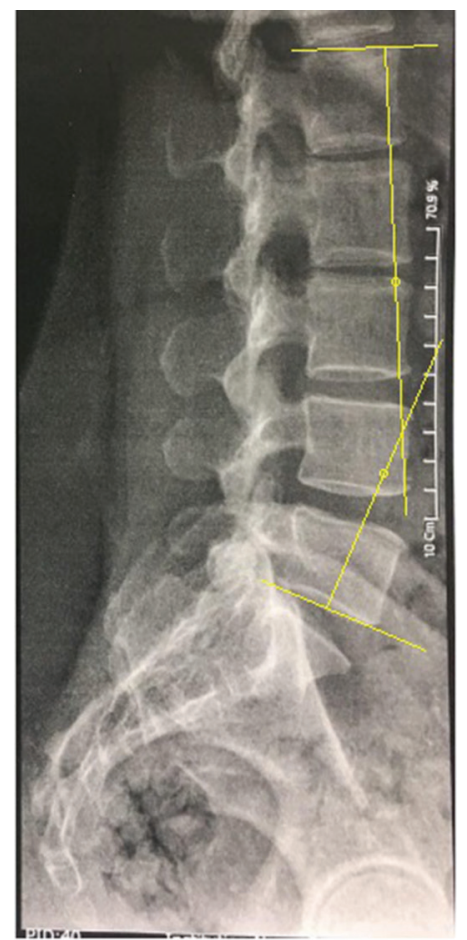

Fig. 2 Measurement of L1-S1 lordosis.

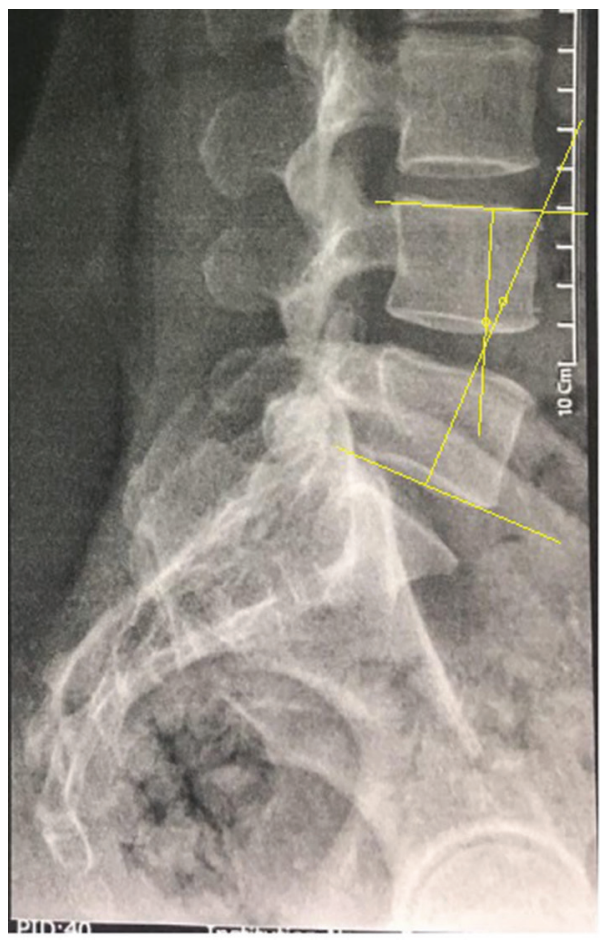

Fig. 3 Measurement of L4-L5 segmental lordosis.

of L3-L4 to L5-S1 on axial planes of computed tomographic (CT) images as presented in - Fig. 4. Existence of osteophytes was considered as the presence of osteoarthritis. We collected all the aforementioned parameters for each patient, and they were statistically analyzed using SPSS software (version 21; IBM Corp.). The significance cutoff was $p>0.05$. 


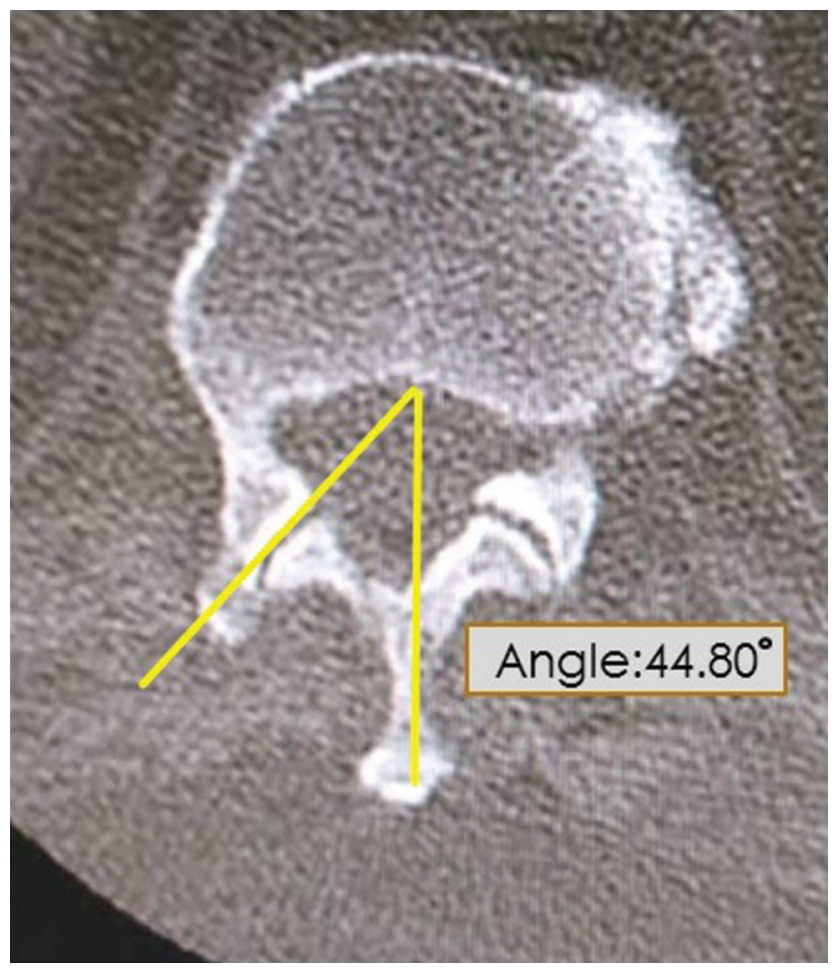

Fig. 4 Measurement of facet joint orientation.

\section{Results}

In this study, we enrolled two groups of patients. DS patients included 36 (60\%) women and 24 (40\%) men with mean age of $52.22 \pm 8.81$ years, and non-DS patients consisted of 24 women (40\%) women and 36 (60\%) men with mean age of $52.85 \pm 10.11$ years. DS patients with average BMI of $29.92 \pm 5$ were significantly obese, compared with non-DS patients with average BMI of $28.29 \pm 4.28$ ( $p=0.031)$.

The radiologic parameters are detailed in Tables $1-3$. As shown in - Table 1, PI, SS, and PT were significantly higher in DS group than in non-DS patients $(p=0.00,0.001,0.04$, respectively). Significant increase in L1-S1 lordosis in DS patients was another finding ( 41.83 vs. $32.92, p=0.001)$.

Comparing segmental lordosis from L1-L2 to L5-S1 levels between the two groups demonstrated a significant difference at more cranial segments (-Table 2). Therefore, that segmental lordosis was significantly higher in DS patients compared with non-DS patients at L2-L3, L3-L4, and L4-L5 levels (7.5 vs. 4.85, $p=0.001,14.03$ vs. $6.78, p=0.00$, 18.31 vs. $14.33, p=0.007$, respectively).

Findings of facet joint orientation in axial CT imaging are described in - Table 3. It is notable that the L3-L4 facet joints are oriented more sagittally in DS patients compared with non-DS group (37.55 vs. $40.71, p=0.01$ ). Nearly $83 \%$ of DS patients had osteoarthritis, but only $30 \%$ of non-DS patients had osteophytes on X-ray.

\section{Discussion}

Spondylolisthesis, characterized by abnormal sacropelvic morphology and orientation, leads to disturbed global sagittal balance of the spine. ${ }^{11}$ In other words, the natural history of spondylolisthesis develops mechanical instability, which

Table 1 Comparison of PI, SS, PT, and L1-S1 lordosis in DS and non-DS patients

\begin{tabular}{|l|l|l|l|}
\hline Sacropelvic alignment parameters & DS (mean \pm SD) & Non-DS (mean \pm SD) & $p$-Value \\
\hline Pelvic incidence & $56.39 \pm 11.51$ & $46.27 \pm 9.84$ & 0.000 \\
\hline Sacral slope & $38.28 \pm 10.32$ & $32.35 \pm 9.16$ & 0.001 \\
\hline Pelvic tilt & $18.52 \pm 9.11$ & $14.02 \pm 7.03$ & 0.04 \\
\hline L1-S1 lordosis & $41.83 \pm 11.49$ & $32.92 \pm 13.62$ & 0.001 \\
\hline
\end{tabular}

Abbreviations: DS, degenerative spondylolisthesis; PI, pelvic incidence; PT, pelvic tilt; SD, standard deviation; SS, sacral slope.

Table 2 Segmental lordosis from L1-L2 to L5-S1 in DS and non-DS patients

\begin{tabular}{|l|l|l|l|}
\hline Sacropelvic alignment parameters & DS (mean \pm SD) & Non-DS (mean \pm SD) & $p$-Value \\
\hline Segmental L1-L2 lordosis & $5.52 \pm 3.75$ & $4.65 \pm 2.77$ & 0.32 \\
\hline Segmental L2-L3 lordosis & $7.50 \pm 4.67$ & $4.85 \pm 3.47$ & 0.001 \\
\hline Segmental L3-L4 lordosis & $14.03 \pm 6.06$ & $6.78 \pm 4.13$ & 0.00 \\
\hline Segmental L4-L5 lordosis & $18.31 \pm 7.88$ & $14.33 \pm 7.20$ & 0.007 \\
\hline Segmental L5-S1 lordosis & $17.79 \pm 8.18$ & $15.08 \pm 7.90$ & 0.052 \\
\hline
\end{tabular}

Abbreviations: DS, degenerative spondylolisthesis; SD, standard deviation.

Table 3 Facet joint orientation of L3-L4 to L5-S1 levels in DS and non-DS patients

\begin{tabular}{|l|l|l|l|}
\hline Facet joint orientation & DS (mean \pm SD) & Non-DS (mean \pm SD) & $p$-Value \\
\hline L3-L4 & $31.87 \pm 6.86$ & $34.47 \pm 6.69$ & 0.06 \\
\hline L4-L5 & $37.55 \pm 7.07$ & $40.71 \pm 6.32$ & 0.01 \\
\hline L5-S1 & $44.67 \pm 8.47$ & $45.88 \pm 7.88$ & 0.53 \\
\hline
\end{tabular}

Abbreviations: DS, degenerative spondylolisthesis; SD, standard deviation. 
advances compensatory responses. ${ }^{2}$ The most commonly used spinal alignment parameters include PI, PT, SS, and lumbar lordosis. ${ }^{8,11}$ Labelle et al found a significant increase in all of these parameters in DS patients, compared with control population. ${ }^{12}$

Roussouly classified sagittal orientation of the lumbar spine and pelvis in four groups. High PI and SS and lumbar lordosis are the main features in type IV of this classification. ${ }^{13}$ Of note, the cause-effect relationship between increased PI and spondylolisthesis is unclear, although the correlation between them has been emphasized in the literature, ${ }^{11}$ even though some studies reported a low PI in DS patients. ${ }^{8}$ Increased mean PI has been suggested in many studies reported as $62.5 \%$ by Morel et al, ${ }^{14} 60 \%$ by Barrey et al, ${ }^{15} 66.2 \%$ by Schuller et al, ${ }^{9}$ and $58.8 \%$ by Ferrero et al. ${ }^{8}$ Our findings were compatible with others in this regard (56.39\%). Average reported PT in Schuller's study was $21 \%^{9}$ and $23.1 \%$ in Ferrero's study. ${ }^{8}$ Our study also showed a significant increase in DS patients but with lower degrees (16.64\%). Morel et al suggested disc degeneration of lower lumbar levels and posterior tilt as the reason. ${ }^{14}$ Schuller et al debated increment in segmental lordosis at the levels above spondylolisthesis, which might lead to posterior stress on facet joints. ${ }^{9}$ They also observed nonsignificant increased lumbar lordosis in comparison with reference group, ${ }^{9}$ but a significant difference was observed between groups in Ferrero's study. ${ }^{8}$ High lumbar lordosis accelerates osteoarthrosis changes..$^{15}$ Contemporary occurrence of vertebral slippage and disc degeneration and collapse also facilitate arthrosis changes of posterior facets. ${ }^{15}$ We found comparable segmental lordosis at L5-S1 levels, but significant increase in L1-L2, L2-L3, and L4-L5 lordosis.

One of the critical roles of facet joints is inhibition of excessive axial rotation. ${ }^{16}$ Therefore, when sagittal orientation of facet joints happens, anterior slippage will occur. ${ }^{17,18}$ In DS patients, elevated sagittal orientation of facet joints at the level of L4-L5 is a feature. ${ }^{9,17,19}$

According to prior investigations, the relationship between overweight and vertical deviation of S1 endplate predisposes L4 to anterior displacement on L5. Jacobsen et al suggested a significant association between overweight and manifestation of DS. ${ }^{20}$ Obesity might lead to more axial load on L4-L5 discs and facet joints, in addition to anterior displacement of the trunk. ${ }^{9}$

Reduction and fixation with circumferential arthrodesis would modify loading on lumbosacral segments. ${ }^{21}$ Restoration of sagittal balance and physiologic lumbar lordosis is the main target of surgical correction..$^{15}$

\section{Conclusion}

Increased PI, PT, and SS are the main features in most DS patients. Increasing segmental lordosis at higher levels is evident in many of these patients. Sagittal facet joint orientation at L4-L5 level is another finding of this study. Notably, this study insists that female sex and obesity are more frequent in this population. Surgical interventions may correct abnormal loading, and consequently would diminish the symptoms.

\section{Ethics}

This research has been approved by ethics committee of Guilan University of Medical Sciences. Written informed consent was obtained from all patients.

\section{Conflicts of Interest}

The authors declare that they have no conflicts of interest.

\section{Acknowledgments}

The authors would like to acknowledge the support of the Neurosurgery Department of Poursina Hospital, Guilan University of Medical Sciences. Also, the cooperation of the physicians and nurses of the neurosurgery wards, Poursina Hospital, Rasht, Iran, is highly appreciated.

\section{References}

1 Mardjetko S, Albert T, Andersson G, et al. Spine/SRS spondylolisthesis summary statement. Spine 2005;30(6, Suppl):S3

2 Winn H.R. Youmans and Winn Neurological Surgery. 7th ed. Philadelphia, PA: Elsevier;2017

3 Alijani B, Emamhadi M, Behzadnia $\mathrm{H}$, et al. Curb the pain of spondylolisthesis; comparing posterolateral fusion with posterior lumbar interbody fusion. Iran J Neurosurg 2015;1(2):22-26

4 Van Schaik JP, Verbiest H, Van Schaik FD. The orientation of laminae and facet joints in the lower lumbar spine. Spine 1985;10(1):59-63

5 Pedram MDR, Vital JM. Spondylolisthesis lombaire degeneratif: Encyclopedie Medico-Chirurgicale. Paris, France: Elsevier;2003

6 Tebet MA. Conceitos atuais sobre equilíbrio sagital e classificação da espondilólise e espondilolistese. Rev Bras Ortop 2014;49:3-12

7 Videbaek TS, Bünger CE, Henriksen M, Neils E, Christensen FB. Sagittal spinal balance after lumbar spinal fusion: the impact of anterior column support results from a randomized clinical trial with an eight- to thirteen-year radiographic follow-up. Spine 2011;36(3):183-191

8 Ferrero E, Ould-Slimane M, Gille O, Guigui P; French Spine Society (SFCR). Sagittal spinopelvic alignment in 654 degenerative spondylolisthesis. Eur Spine J 2015;24(6):1219-1227

9 Schuller S, Charles YP, Steib J-P. Sagittal spinopelvic alignment and body mass index in patients with degenerative spondylolisthesis. Eur Spine J 2011;20(5):713-719

10 Layegh M, Hejazian E. Prevalence of spondylolysis and spondylolisthesis in patients afflicted with chronic back pain in Babol City, Iran, during 2012 and 2013. Iran. J Neurosurg 2017;3(1):8-14

11 Labelle H, Mac-Thiong J-M, Roussouly P. Spino-pelvic sagittal balance of spondylolisthesis: a review and classification. Eur Spine J 2011;20(Suppl 5):641-646

12 Labelle H, Roussouly P, Berthonnaud E, et al. Spondylolisthesis, pelvic incidence, and spinopelvic balance: a correlation study. Spine 2004;29(18):2049-2054

13 Roussouly P. Influence de l'organisation morphologique sagittale de l'ensemble rachis-bassin sur l'évolution des pathologies dégénératives rachidiennes. Cahiers d'enseignement de la SOFCOT. In: Vital J. Alternatives à l'arthrodèse lombaire et lombosacrée. Paris, France: Elsevier;2007:27-35

14 Morel E, Ilharreborde B, Lenoir T, et al. Analyse de l'équilibre sagittal du rachis dans les spondylolisthésis dégénératifs. Rev Chir Orthop Repar Appar Mot 2005;91(7):615-626

15 Barrey C, Jund J, Perrin G, Roussouly P. Spinopelvic alignment of patients with degenerative spondylolisthesis. Neurosurgery 2007;61(5):981-986, discussion 986 
16 Adams MA, Hutton WC. The relevance of torsion to the mechanical derangement of the lumbar spine. Spine 1981;6(3):241-248

17 Boden SD, Riew KD, Yamaguchi K, Branch TP, Schellinger D, Wiesel SW. Orientation of the lumbar facet joints: association with degenerative disc disease. J Bone Joint Surg Am 1996;78(3):403-411

18 Sharma M, Langrana NA, Rodriguez J. Role of ligaments and facets in lumbar spinal stability. Spine 1995;20(8):887-900

19 Toyone T, Ozawa T, Kamikawa K, et al. Facet joint orientation difference between cephalad and caudad portions: a possible cause of degenerative spondylolisthesis. Spine 2009;34(21):2259-2262

20 Jacobsen S, Sonne-Holm S, Rovsing H, Monrad H, Gebuhr P. Degenerative lumbar spondylolisthesis: an epidemiological perspective: the Copenhagen Osteoarthritis Study. Spine 2007;32(1):120-125

21 Muñiz Luna L, Vilchis Sámano H, Marban Heredia M, et al. Functional disability, sagittal alignment and pelvic balance in lumbar spondylolisthesis. Coluna/Columna 2016;15(1):30-32 